\title{
Identification of Methylation-Driven Genes Prognosis Signature and Immune Microenvironment in Epithelial Ovarian Cancer
}

\section{Mingzi Tan}

Liaoning Provincial Tumor Hospital: Liaoning Cancer Institute and Hospital

\section{Shengtan Wang}

Hainan General Hospital

Feifei Li

Shandong Provincial Hospital

Haoya Xu

Shengjing Hospital of China Medical University

Jian Gao

Shengjing Hospital of China Medical University

Liancheng Zhu ( $\sim$ zhulc@sj-hospital.org )

Shengjing Hospital of China Medical University https://orcid.org/0000-0002-3966-8425

\section{Primary research}

Keywords: ovarian cancer, DNA methylation-driven genes, immune microenvironment, risk score, nomogram, prognostic model

Posted Date: October 13th, 2021

DOl: https://doi.org/10.21203/rs.3.rs-910488/v1

License: (c) (i) This work is licensed under a Creative Commons Attribution 4.0 International License. Read Full License 


\section{Abstract \\ Background}

Aberrant gene methylation has been implicated in the development and progression of tumors. We aimed to identity methylation driven genes involved in EOC to establish a prognostic signature for patients with EOC.

\section{Methods}

The methylation, RNA expression and clinical data of EOC patients were downloaded from UCSC Xena website. The "MethylMix" R package was employed to identify the methylation driven genes (MDGs), GO and KEGG analyses were performed to identify the biological functions of the identified genes, univariate, LASSO, and multivariate analyses were carried out to reveal key prognostic genes. Combined with clinical parameters, a prognostic risk nomogram model was established, and its predictive value was internally evaluated using AUC curves and externally verified in the GEO database.

\section{Results}

111 differentially expressed MDGs were identified, among which six (SLC9A1, MARVELD1, PI3, MFAP4, MSX1, IL18BP) were selected to establish a prognostic risk scoring model. Results of univariate and multivariate analyses indicated that the risk score model was an independent risk factor for EOC. Combined with clinical parameters, a nomogram was constructed, which showed good predictive performance as evidenced by AUC values. The risk scores and the nomogram were externally verified using GSE26193 and GSE53963 datasets. Analysis of mRNA levels of the six genes in OVCAR3 before and after DAC treatment revealed that DAC inhibited the methylation of six genes, thereby increasing mRNA levels of the six genes. GSEA analysis show that associated with the 6 MDGs in the two risk score model groups signaling pathways are closely related to tumor invasion and metastasis. There are significant differences between the two risk score model groups in terms of immune function, check point and TMB.

\section{Conclusions}

We identified and verified 6 MDGs that are closely related to the prognosis of ovarian cancer. A prognostic risk score model and nomogram for predicting the prognosis of EOC were constructed based on the six MDGs. It can also effectively reflect the immune environment and immunotherapy response of EOC. These MDGs have great significance to the implementation of individualized treatment and disease monitoring of EOC patients. 


\section{Introduction}

The mortality rate of epithelial ovarian cancer (EOC) ranks first among the three types of malignant tumors in the female reproductive system[1]. Due to its specialized anatomical location, nearly $80 \%$ of EOC are already at an advanced stage at the time of diagnosis[2]. The incidence of EOC has declined, however, the age of onset has also decreased, posing a serious threat to the life and fertility of women of childbearing age [1]. The standard treatment of EOC is mainly surgery, followed by platinum-based chemotherapy[3]. However, due to the emergence of chemotherapy resistance, the 5-year survival rate of advanced EOC is only 15-20\% [1]. Early detection and early treatment have a significant impact in reducing the mortality of EOC patients and improving the survival rate. Therefore, the establishment of an effective assessment and prediction system for survival outcomes in patients with EOC can effectively improve the treatment effect and quality of life.

DNA methylation is one of the highly tissue-specific epigenetic modifications that can maintain and stabilize the expression of tissue-specific genes [4]. Methyltransferase regulates the degree of DNA methylation by adding or removing methyl groups on DNA, thereby affecting DNA transcription and expression. The expression of methylation-modified genes changes dynamically. Studies have shown that hyperhomocysteinemia may change the status of DNA methylation and cause changes in intracellular signaling pathways and thus cause skeletal muscle diseases[5]. Hypermethylation inhibits gene expression, while demethylation promotes gene expression. Therefore, methylation-regulated gene expression, including oncogene and tumor suppressor genes, plays a double-sided role in EOC development [6]. Previous studies report that hypermethylation is associated with the inactivation of almost all pathways involved in the occurrence and development of EOC [7-12]. At present, numerous studies have used the MethylMix R package [13] to screen Methylation-driven genes (MDGs), explore possible markers, establish related models, and predict the correlation between the target gene methylation level and diagnosis, survival, and recurrence of malignant tumors, which can help clarify the occurrence and development of malignant tumors. To explore possible diagnostic and prognostic tumor markers, Lv et al. identified four genes in thyroid cancer whose changes in methylation levels were closely related to poor prognosis [14]. In another study, two MDGs (SPP1 and LCAT) were screened out as independent risk factors for poor prognosis, and tumor recurrence of liver cancer [15]. Besides, there are also other related studies reported in lung cancer [16], kidney cancer [17], head and neck squamous cell carcinoma [18], and other malignant tumors. However, no related research has been reported in EOC.

Therefore, this study used The University of California Santa Cruz (UCSC) Xena online data to comprehensively analyze and identify EOC MDGs, construct a risk score prognostic model for EOC patients, and establish a nomogram with MDGs and clinicopathological risk factors to predict the overall survival (OS) of patients with EOC. The establishment of an effective prediction system for assessing the survival outcomes in ovarian cancer (OC) patients can improve the treatment effect and quality of life. Changes in the immune microenvironment play an important role in the progression of ovarian cancer and are also an important target for ovarian cancer treatment[19]. Studies have shown that immunomethylation analysis can effectively screen tumor immunotherapy options and evaluate tumor 
prognosis[20]. Therefore, we further grouped them according to the risk score constructed by MDGs, analyzed the correlation with the immune pathway of ovarian cancer, and provided new ideas for ovarian cancer immunotherapy.

\section{Methods}

\section{Patients and samples}

A total of 380 OC RNA-sequencing profiles, 616 DNA methylation profiles (602 OC and 14 normal ovarian) and associated clinical data of EOC patients were downloaded from the UCSC Xena website (https://xena.ucsc.edu/) (up to December 1, 2020). Among the 372 EOC samples (RNA-sequencing data and paired DNA methylation data), 335 had overall survival (OS) data. To externally validate the risk score model and nomogram, GSE26193 (107 OC samples, including 87 EOC)[21], GSE53963 microarray dataset (174 high-grade serous ovarian carcinoma samples and a reference pool of 106 ovarian samples)[22], and associated clinical data from the Gene Expression Omnibus (GEO) database were downloaded.

\section{Identification of DNA MDGs in EOC}

The MethyIMix R package was used to analyze methylation and RNA-seq data, determine the correlation between the degree of methylation and gene expression, and screen for genes that are significantly related to hypermethylation and hypomethylation, to obtain MDGs for EOC.

\section{Functional Annotation and Enrichment Analysis of DNA MDGs}

A total of 335 DNA MDGs were subjected to Gene Ontology (GO) analysis in R software. To perform functional and pathway enrichment analysis, Kyoto Encyclopedia of Genes Genomes (KEGG) pathway enrichment analysis and gene annotation analysis for DNA MDGs were used in Metascape online (http://metascape.org/). Enriched ontology clusters were colored by cluster-ID.

\section{Survival Analysis of Candidate MDGs}

Survival R software package was used to analyze the correlation between the expression of the prognostic-related genes in EOC and the degree of methylation. To analyze the relationship between the OS of EOC patients and MDGs, Kaplan-Meier (KM) analysis and univariate COX analysis were performed.

\section{Construction and Validation of the Predictive Model}

To further screen for MDGs related to prognosis in EOC, we used univariate Cox and multivariate Cox analysis, and the least absolute shrinkage selection operator (LASSO) to construct a risk score model.EOC patients were divided into the low-risk and high-risk groups based on the risk score. The X-tile software was used to find the optimal critical value for grouping. KM analysis was used to compare the 
survival rate difference in the two groups. Time-dependent area under the curve (AUC) analysis was performed to evaluate the predictive performance of the model.

\section{Predictive factors among the Clinicopathological Characteristics}

Univariate and multivariate Cox regression analyses were performed to determine whether the six DNA MDG-based risk scores were independent risk factors of EOC. Besides, a stratified analysis was carried out based on the clinical-pathological characteristics of the patients, including age, race, FIGO stage, lymphatic invasion, histological type, and tumor residual. GSE26193 and GSE53963 cohorts were used to validate the predictive model.

\section{Construction and verification of the Nomogram}

The nomogram was generated by the "rms" package based on age, FIGO stage, and risk score. Timedependent AUC and concordance index (C-index) were used to evaluate the nomogram. The Decision curve analysis (DCA) was used to quantify the clinical utility based on the clinical consequences. GEO cohort GSE53963 was used to verify the nomogram.

\section{Copy Number Variation (CNV), Mutation Characteristics, and Gene Set Enrichment Analysis (GSEA) of the Six DNA MDGs}

Graphic illustrations of CNV and the six-gene mutation profiles in all EOC tissues from the UCSC Xena dataset were obtained from cBioPortal(http://www.cbioportal.org/). GSEA was performed using the gsea3.0.jar software (http://software.broadinstitute.org/gsea/index.jsp).

\section{Cell culture}

The EOC cell line OVCAR3 was purchased from the National Collection of Authenticated Cell Cultures. The cell line is free of mycoplasma contamination and the cells were grown in RPM1640 medium (Biological Industries) supplemented with 10\% fetal calf serum (Biological Industries) and cultured at $37^{\circ} \mathrm{C}$, in $5 \% \mathrm{CO}_{2}$. To the cell medium, $1 \mu \mathrm{M}[2], 10 \mu \mathrm{M}$ 5-aza-2'-deoxycytidine (DAC) (Sigma) was added, cultured for $72 \mathrm{~h}$, and the cell culture medium was changed every day. The cells were harvested for mRNA expression analysis of the candidate gene.

\section{Validation of the mRNAs expression of the six genes}

Total mRNA was extracted from OVCAR3 cells using Trizol (TaKaRa). cDNA was reverse transcribed using the PrimeScript RT reagent Kit with gDNA Eraser (TaKaRa). Real-time-PCR analysis was performed using the SYBR-Green method. The primers used for Real time-PCR are shown in Table S3. The relative expression of mRNAs was calculated using the $2^{-\Delta \Delta C t}$ method.

\section{Immune assessment of two risk score model groups}


We use the Infiltration Estimation software in time 2.0 (http//: www.timer.comp-genomics.org) to perform Immune related heat map analysis of the two risk score groups. Immune function was analysis by ssGSEA algorithm,together with Check point to study the correlation between MDGs and immune phenotypes.VarScan2 was used to analysis the tumor cell mutations to reveal the correlation between the immunotherapy response and the two risk score model groups.

\section{Statistical Analysis}

All statistical analyses were performed in the R software and the tests were two-sided. $\mathrm{P}$ values less than 0.05 were considered to be statistically significant.

\section{Results}

\section{Clinicopathological Characteristics of Enrolled OC Patients}

The study flowchart is shown in Fig. 1. A total of 335 EOC methylation-related data samples were obtained from the UCSC Xena website. The median age of the patients was 59 years (30 to 87 years), while the median overall survival was 1039 days (31-5481 days). There were 20 cases of stage I-II, 315 cases of stage III-IV, and 83 cases of lymphatic metastasis. The clinicopathological features of the 335 patients are listed in Table 1. 
Table 1

The clinicopathological features of patients $(\mathrm{N}=335)$

\begin{tabular}{|ll|}
\hline characteristics & number (\%) \\
\hline Age & \\
\hline$\geq 65$ & $105(31.3)$ \\
\hline Race & $230(68.7)$ \\
\hline White & \\
\hline Black and African American & $17(5.1)$ \\
\hline Others & $22(6.5)$ \\
\hline FIGO stage & \\
\hline I-II & $20(6.0)$ \\
\hline III- IV & $315(94)$ \\
\hline Histologic grade & \\
\hline $1-2$ & $53(12.8)$ \\
\hline $3-4$ & $292(87.2)$ \\
\hline Lymphatic invasion & \\
\hline NO & $252(75.2)$ \\
\hline YES & $83(24.7)$ \\
\hline Tumor residual & \\
\hline NO* & $23(6.9)$ \\
\hline $1 \sim 2 c m$ & $36(10.7)$ \\
\hline$>2 c m$ & \\
\hline others & \\
\hline * No Macroscopic disease & \\
\hline
\end{tabular}

\section{Dna Mdgs In Oc}

The MethyIMix R package identified 111 DNA MDGs of OC, including 50 hypermethylated and 61 hypomethylated genes (Table S1). 


\section{Enrichment analysis}

We screened 111 MDGs (P-value < 0.05), and the level of gene methylation was visualized via heatmap (Fig. 2A). The "clusterProfiler" and "GOplot" package were used to perform GO enrichment analysis to determine the function of the MDGs in OC. The identified MDGs were found to be closely related to "CELLULAR RESPONSE TO NICOTINE", "POSITIVE REGULATION OF ION TRANSMEMBRANE TRANSPORTER ACTIVITY”, "TRANSEPITHELIAL WATER TRANSPORT”, "GRANULOCYTE MACROPHAGE COLONY - STIMULATING FACTOR BIOSYNTHETIC PROCESS", etc (Fig. 2B). The top 20 clustered KEGG pathway is shown in Fig. $2 \mathrm{C}$ and was converted into a network based on Kappa-statistical similarities and visualized by Cytoscape (v.3.1.2) (Fig. 2D). The MDGs were found to play an important role in the "HALLMARK ESTROGEN RESPONSE LATE”, "IL-18 signaling pathway”, “PID PDGFRB PATHWAY”, "MAPK signaling pathway" and other important pathways involved in tumorigenesis and development.

\section{Prognostic Value Of Dna Mdgs In Eoc}

Univariate analysis was used to screen for survival-related MDGs, and a total of 14 MDGs were identified, including MARVELD1, MFAP4, EMILIN1, PI3, SLC9A1, IL18BP, FBLN2,SCTA5A, SCNN1A, GSTP1, RPL39LI MSX1, CD3G, ACTA2 $(\mathrm{P}<0.05)$ (Table S2). KM analyzed the correlation between the expression of the 14 genes and OS of EOC patients (Figure S1A). LASSO regression was used to narrow down the range of target genes (Figure S1B). A total of twelve DNA MDGs (EMILIN1, FBLN2, STAT5A, SCNN1A, GSTP1, CD3G, SLC9A1DMARVELD1, PI3, MFAP4, MSX1, IL 18BP) were identified as independent predictive factors (Figure S1C). COX multivariate analysis was performed to determine the correlation between the mRNA expression of the twelve genes and OS of EOC patients, as well as the correlation between the level of DNA MDGs and OS of EOC patients. Finally, six DNA MDGs (SLC9A1DMARVELD1, PI3, MFAP4, MSX1, IL 18BP) were used to construct the risk score model (Table 2), among them SLC9A1DMARVELD1DPI3 and MFAP4 were highly expressed and hypomethylated, while MSX1, IL 18BP were lowly expressed, and their hypermethylation closely related to poor prognosis of EOC (Fig. 3A, C). The DNA methylation level was found to be closely related to mRNA expression $(|R|>0.3, P<0.05)$ (Fig. 3B). 
Table 2

Results of the six genes in the multivariable Cox regression analysis.

\begin{tabular}{|llllll|}
\hline Genes & Coefficient & HR & HR.95L & HR.95H & $P$ value \\
\hline SLC9A1 & 0.063 & 1.065 & 1.024 & 1.108 & 0.002 \\
\hline MARVELD1 & 0.024 & 1.025 & 1.000 & 1.049 & 0.046 \\
\hline PI3 & 0.013 & 1.014 & 1.007 & 1.020 & $2.511 \mathrm{E}-05$ \\
\hline MFAP4 & 0.013 & 1.013 & 1.001 & 1.025 & 0.031 \\
\hline MSX1 & -0.015 & 0.985 & 0.973 & 0.997 & 0.017 \\
\hline IL18BP & -0.105 & 0.900 & 0.844 & 0.960 & 0.001 \\
\hline HR: hazard ratio; L: low; H: high. & & & \\
\hline
\end{tabular}

\section{Construction Of A Prognostic Risk Score Model For Eoc}

Complete clinical and expression data of the six genes (SLC9A1,MARVELD1, PI3, MFAP4, MSX1, IL 18BP) in EOC patients were used to calculate the risk score. The X-tile chart was used to determine the optimal cutoff value. $\mathrm{KM}$ analysis revealed that the OS of the high-risk score group was remarkably shorter than that of the low-risk score group (Fig. 4A). The distribution of the six gene signature risk scores is shown in Fig. 4B and a heatmap was used to show the expression profiles of the six genes and the related risk scores (Fig. 4D). Univariate and multivariate analyses of age, race, FIGO stage, lymphatic invasion, histologic grade, tumor residual, and risk score were performed. Univariate analysis showed that age, FIGO stage, and risk score were independent risk factors for poor prognosis of EOC patients, while multivariate analysis showed that age and risk score were independent risk factors for poor prognosis of EOC patients (Fig. 4C).

\section{Construction And Evaluation Of The Nomogram}

The area under the curve (AUC) for 3, 5, 10-years OS demonstrated the prognostic power of the risk score signature. The AUCs for 3-, 5-and 10-year survival times were $0.673,0.696$, and 0.801 , respectively, and were significantly higher than the prognostic power of age and FIGO stage (Fig. 5A). The time-dependent AUC of the risk score was found to be significantly higher than that of age and FIGO stage for the 10 years OS prediction (Fig. 5B),indicating favorable discrimination based on the risk score. We constructed a nomogram for predicting the individualized probability of EOC survival times in clinical practice, taking into consideration, age, FIGO stage, and risk score (Fig. 5C), then the clinical model was assessed by DCA. The results showed that the nomogram performed better compared with the age or FIGO stage model. Therefore, the constructed nomogram can be used by clinicians as a prognostic guide to make accurate clinical decisions for EOC patients (Fig. 5D). The calibration curve of the nomogram at 3, 5, 10 years OS were close to $45^{\circ}$ line】thus, demonstrated high predictive accuracy (Fig. 5E). 


\section{External Validation of the nomogram}

For further external validation, we validated the constructed 6-methylation-driven gene-based risk score prediction model in the GSE26193 dataset. The results of KM analysis suggested that the high-risk group had a poor prognosis ( $p<0.05$, Fig. $6 \mathrm{~A}$ ). The AUCs for $0.5-, 1$-and 3-year survival times in GSE26193 were $0.873,0.852$, and 0.715 (Fig. 6B). Compared with stage and grade, risk score had the highest AUC value at three years $(0.5,1,3$ year), an indication that the best performing model was based on the risk score model in the validation dataset (Fig. 6C). In the GSE53963 dataset, KM analysis revealed that EOC patients with high-risk scores or high nomogram risk score $(P<0.01)$ had a poor prognosis (Fig. 6D, E). Furthermore, there were significant differences in prediction outcomes between FIGO staging, histologic grade, and risk-score (Fig. 6F). The AUCs of the risk-score and nomogram score at 0.5-, 1-, 5-and 10-year survival times were $0.744 / 0.645,0.691 / 0.645,0.685 / 0.645$, and $0.746 / 0.645$, respectively, which were significantly higher than age and histologic grade (Fig. 6G), similarly to the time-dependent AUC (Fig. 6H). Based on the validation results, the nomogram was found to be more accurate in predicting short-term and long-term prognosis of EOC than risk score, age, and FIGO staging. Therefore, the constructed nomogram can be effective in making accurate clinical decisions and follow-up for EOC patients.

\section{Cnv, Mutation Characteristics And Pathway Enrichment Analysis}

To study the effect of methylation on the expression of the selected genes, CNV analysis was performed to determine the factors affecting gene transcription. The results showed that transcription of the six genes (SLC9A1,MARVELD1, PI3, MFAP4, MSX1, IL 18BP) was affected by gene amplification, deletion, and mutation. After eliminating these effects, changes in the degree of methylation had a significant effect on the transcription and expression level of the 6 candidate genes. These results showed that DNA MDGs play a key role in EOC. GSEA was used to investigate the signaling pathways associated with the 6 MDGs in the two risk score model groups. In the high-risk score group, the top five signaling pathways (risk score > 0.314, Fig. 7B) were "ADHERENS JUNCTION", "NOTCH SIGNALING PATHWAY", "ERBB SIFNALING PATHWAY", "FOCAL ADHESION", "HEDGEHOG SIGNALING PATHWAY", while in the low-risk score group, the top five signaling pathways (risk score < 0.314, Fig. 7C) were "PROTEASOME", "OXIDATIVE PHOSPHORYLATION”, "AUTOIMMUNE THYROID DISEASE”, "PRIMARY IMMUNODEFICIENCY” and "PARKINSONS DISEASE". Most of these signaling pathways are closely related to tumor invasion and metastasis and promote the development of tumors.

\section{9. mRNA Expression of the six genes in OVCAR3 cells after DAC treatment}

Studies have shown that the six genes (SLC9A1,MARVELD1, PI3, MFAP4, MSX1, IL 18BP) play an important role in the development of EOC. To determine the effect of methylation on the expression of the 
six genes, EOC cell line OVCAR3 was treated with DAC. DAC was found to inhibit methylation in the six genes, thus, increasing the mRNA expression levels(Fig. 8).

\section{Immune Assessment Of Two Risk Score Model Groups}

Our analysis found that the TMB level of the high risk group was lower than that of the low risk group ( $P$ $<0.049$ ), which was negatively correlated with the risk score (Fig. 9A, B). The attached figure shows the results of somatic mutations in the high risk group (Figure S2A) and the low-risk group (Figure S2B). Further analysis showed that DNAH11, CHD4, and CREBBP have a higher mutation frequency in the low risk group, while BRCA1, MYH1, and MDN1 have a higher mutation frequency in the high risk group (Figure S2C). We use heat maps to show the analysis of TIMER, CIBERSORT, CIBERSOTR-ABS, QUANTISEQ, MCPCOUNTER, XCELL, EPIC of high-risk groups and low-risk groups (Fig. 9C). Among them, 16 groups with significant differential expression in TIMER, CIBERSORT and XCELL were show in Fig. 9D. Figure $9 \mathrm{E}, \mathrm{F}$ shows the correlation between MDGs and Immune function and Check point. We found that compared with the high risk group, MHC-1 factor is high expressed in low risk group while the expression of tumor immune factors LAG3, CD274 (PD-L1) increased significantly. It shows that the risk score model we have established can effectively reflect the immune environment and immunotherapy response of ovarian cancer.

\section{Discussion}

EOC has a high mortality, low survival, and high recurrence rates, significantly affecting the quality of life of EOC patients. Besides, it is difficult to detect EOC at an early stage, which is also attributed to the lack of specific and sensitive biomarkers for predicting the prognosis of EOC patients. In tumor cells, alterations in the epigenome can be detected and have been associated with certain tumor characteristics, such as oncogenic transformation and tumor metastasis [23]. Hypermethylation in EOC is related to the inactivation of almost all pathways involved in EOC development, such as DNA repair, cell apoptosis, and adhesion [7-12]. Studies have shown that DNA methylation changes in circulating blood can be used to detect and predict early-stage EOC [24]. Numerous studies have performed a comprehensive multi-omics analysis of EOC genomics, epigenomics, and transcriptomics, and suggested that gene methylation plays an important role in EOC development [25]. Therefore, the exploration and validation of DNA MDGs in EOC is necessary for early diagnosis and prognosis. With the recent development in sequencing technology, methylation-related epigenetic changes are easy to monitor. Therefore, MethyIMix [13] and UCSC Xena website database were used in the present study to analyze the methylation array and expression profile data of EOC tissue samples and identify abnormal EOC DNA MDGs. Based on the MDGs signature,we constructed and validated a prognostic risk score model, which was then combined with age and FIGO staging to construct a nomogram for predicting the prognosis of EOC patients. 
In our risk model, six DNA MDGs, including SLC9A1,MARVELD1, PI3, MFAP4, MSX1, and IL 18BP were selected and used to calculate the risk score. Among the six DNA MDGs, SLC9A1 (SoLute Carrier family 9A1) [26] gene encodes Sodium/hydrogen exchanger 1 (NHE1), the main $\mathrm{H}^{+}$efflux mechanism in maintaining alkaline pHi in human cancer cells [27]. Consistent with the results of this study, previous studies have shown that high expression of SLC9A1 (NHE1) is associated with a decrease in the overall survival of OC patients since NHE1 promotes the proliferation of OC cells. Cancer cells secrete protons $(\mathrm{H}$ + ) into the extracellular medium through the $\mathrm{Na}+\mathrm{H}+$ exchanger 1 (NHE1) to adjust the intracellular $\mathrm{pH}$ value and promote tumor proliferation. Increased expression and activity of NHE1 predict poor prognosis in EOC patients [28]. MARVEL are myelin and lymphocyte-associated proteins (MAL) and related proteins for vesicle trafficking and membrane link. MARVELD1 is a member of the MARVEL domain-containing proteins. The expression of MARVELD1 is regulated by DNA methylation in multiple cancers. Some scholars have found that MARVELD1 is lowly expressed in OC, and maybe a tumor suppressor gene for OC [29]. Other studies on high-throughput chips (1347) and expanded samples (81 cases) have shown that high expression of MARVELD1 in OC samples increases chemoresistance to platinum and taxane combination therapy [30]. In this study, low methylation and high expression were found to be unfavorable factors for prognosis in OC. Therefore, high expression of MARVELD1 predicts a poor prognosis for OC by promoting chemotherapy resistance. MFAP4 is one of five MFAPs family members and plays significant biological roles in human cancers [31-34]. The expression levels of MFAP4 in different tumor types and tumor stages are not consistent. MFAP4 is downregulated in prostate cancer and urinary bladder cancer [35]. However, high expression of MFAP4 in neuroblastoma patients correlates with poor survival [36]. In low-risk breast cancer, MFAP4 protein is reported to be highly expressed [37], while in advanced breast cancer, high expression of MP4 is associated with poor prognosis, and hypermethylation may be a key drive for low MFAP4 transcription and expression [38]. MFAP4 promotes the recruitment of inflammatory cells and strengthens immune monitoring of tumor cells, thereby inhibiting the early occurrence of tumors [32]. Besides, MFAP4 accelerates tumor exacerbation by promoting cancer cell proliferation and migration, when the immune system is fully suppressed in latestage tumors [34]. In summary, the expression level of MFAP4 is regulated by methylation, and plays different biological roles in tumor development., thereby affecting tumor prognosis. The present study for the first time revealed that low methylation and high expression of MFAP4 are poor prognostic factors for OC. However, the role of MFAP4 in the development of OC needs to be studied further. PI3/Elafin (Peptidase Inhibitor 3 ) is an endogenous serine protease inhibitor involved in epithelial protection and is highly expressed in tissues during an inflammatory response [39], and its activity may influence the resolution of inflammation and preserving tissue integrity [40]. Abnormal expression of PI3/Elafin has been detected in a variety of tumors. Elafin is highly expressed in glioblastoma [41] and lowly expressed in breast tumors [42]. Elafin is downregulated during ovarian tumorigenesis, however, it is upregulated with further development of the tumor which is significantly associated with higher tumor stage, ascites, resistance to treatment, and higher serum CA125 [42]. Elafin increases cellular proliferation in OC, and overexpression of Elafin is associated with poor outcomes in OC patients [43]. The present study showed that low methylation and high expression of Elafin drives poor outcome in OC. Elafin is regulated by methylation during OC development. MSX1 (Msh homeobox 1) gene acts as a transcriptional repressor 
during embryogenesis [44]. In previous studies, aberrant methylation of MSX1 promoter DNA has been linked with lung cancer, gastric cancer, Wilms tumor, childhood acute T-lymphoblastic leukemia, and breast cancer [45-49]. MSX1 induces G0/G1 arrest and apoptosis in cervical cancer [50]. Methylation regulation of MSX1 is associated with platinum-resistant disease in high-grade serous EOC and MSX1 over-expression leads to cisplatin sensitization [51]. In this study, MSX1 over-expression and low methylation were found to drive good outcomes in OC. These findings suggest that MSX1 may be a potential tumor suppressor gene, which can inhibit tumor proliferation and enhance the sensitivity of tumor cells to chemotherapeutic drugs. IL-18 is a pleiotropic, proinflammatory cytokine with dual effects on tumor development and progression [52]. IL-18 binding protein (IL-18BP) possesses a high affinity to IL-18, thus, preventing the binding of mature IL-18 to its receptor and inhibits the biological activity of IL18 [53]. Previous studies have found tumor cells and tumor-related white blood cells produce IL-18BP. High levels of IL-18BP in the tumor microenvironment may limit endogenous or therapeutic IL-18 to induce an effective immune response [54]. IL18 induces Th- 2 immune responses, inhibits the recognition of cancer cells by antigen-presenting cells, and promotes immune escape and tumor cell proliferation. Besides, it also promotes the expression of vascular adhesion molecules and angiogenic factors to promote tumor cell invasion and metastasis [55]. The present study found that low methylation and high expression of IL18BP is a favorable prognostic factor in patients with OC. Therefore, IL18BP inhibits further development of $\mathrm{OC}$ by inhibiting tumor proliferation and invasion and also plays an important immune protection role in suppressing OC.

GSEA KEGG pathway enrichment analysis revealed that the top five signaling pathways in the high-risk score group were "ADHERENS JUNCTION"[56], "NOTCH SIGNALING PATHWAY"[57], "ERBB SIFNALING PATHWAY"[58], "FOCAL ADHESION"[59], which are all classic pathways reported in malignant tumor research and play an important role in tumor development.

We screened out $6 \mathrm{MDG}$ s to construct risk score model. Among them, $3 \mathrm{MDG}$ s are closely related to immunity. The immune environment is of great significance in the development and treatment of ovarian cancer. Therefore, we further grouped according to the risk score and analyzed its correlation with the immune pathway of ovarian cancer. Our research found that compared with the low-risk group, the expression of MHC-1 molecules in the high-risk group was significantly reduced. The expression of MHC1 molecules is reduced in $40-90 \%$ of malignant tumors, which is related to poor prognosis. The low expression of MHC-1 molecules can reduce the presentation of antigens, which can cause $\mathrm{CD} 8+\mathrm{T}$ cell recognition and reduce cytotoxicity, promote tumor progression[60]. In the immune checkpoint analysis, we found that LAG3 and CD274 (PD-L1) were highly expressed in the low-risk group. LAG3, CD274 (PDL1) are immunosuppressive factors, and they are also indicators of anti-tumor immunotherapy. CD274 (PD-L1) is a negative regulator of T cells, expressed by tumor-associated macrophages in ovarian cancer, and is positively correlated with the prognosis of ovarian cancer [61]. LAG3 is expressed on the surface of activated T cells and NK cells [62] [63]. The prognostic value of LAG3-positive cells is inconsistent in different types of cancer. In ovarian cancer, the positive expression of LAG3 can improve the poor prognosis of ovarian cancer [64]. We are more surprised to find that the established risk score model is closely related to the level of TMB and can effectively reflect the responsiveness of tumor 
immunotherapy. TMB refers to the number of somatic mutations in the tumor genome after germline mutations have been removed and is a biological indicator that guides immunotherapy. The higher level of TMB, the more new antigens that can be recognized by $T$ cells produced, the better the efficacy of immune checkpoint inhibitor therapy may be, and the better the effect of tumor immunotherapy. However, because of the high cost of TMB analysis, there are certain difficulties in clinical implementation. The model we built is negatively correlated with TMB, which can effectively reflect the level of TMB. In summary, the risk score model related to MDGs we constructed can also effectively reflect the immune environment and immunotherapy response of ovarian cancer.

This study, for the first time, established a prognostic model for OC based on MDGs. Although the established nomogram also included age and FIGO staging, it was found to successfully predict the prognosis of $\mathrm{OC}$ patients and effectively reflect the immune environment and immunotherapy response of ovarian cancer. However, the proportion of hierarchical data is not balanced. OC is most advanced when it is discovered, so the cases we collected accounted for most patients with advanced stages (stage III and IV accounted for 94\%). Previous studies have reported that MDGs can inhibit cancer in the early stage and are affected by the tumor microenvironment in the late stages, thus, promoting cancer development. Besides, in invading tumors, MDGs can regulate DNA methylation. Qualitatively modified genes also frequently undergo mutations. During tumor development, the tumor interacts with the tumor microenvironment, tumor cells acquire phenotypic plasticity, and further promote tumor invasion and metastasis through dynamic phenotypic changes [65]. Therefore, epigenetic modification of tumor cells occurs during tumor development. There are many pathological types of epithelial ovarian cancer, but this study only included serous ovarian carcinoma. Methylation is highly tissue-specific [4], therefore, methylation of MDGs in other pathological types of OC needs to be studied further. In summary, samples with complete clinical information and sequencing-related data will be further expanded in the future, thus, better prognostic nomographs of $\mathrm{OC}$ will be established following careful stratification analysis.

\section{Abbreviations}

AUC: Area under the curve; C-index: Concordance index; CNV: copy number variation; DAC:5-aza-2'deoxycytidine;DCA:decision curve analysis; EOC: epithelial ovarian cancer; FIGO: International Federation of Gynecology and Obstetrics; GEO: Gene Expression Omnibus; GO: Gene Ontology analysis; GSEA: gene set enrichment analysis; HR: hazard ratio; KEGG: Kyoto Encyclopedia of Genes and Genomes; KM: Kaplan-Meier; LASSO: Least absolute shrinkage and selection operator; MDGs: methylation driven genes; OC: ovarian cancer; OS: overall survival; TCGA: The Cancer Genome Atlas; TMB: tumor mutation burden

\section{Declarations}

Ethics approval and consent to participate: All analyses were conducted in accordance with relevant regulations and guidelines.

Consent for publication: All authors agree to publish 


\section{Data Availability}

The gene expression and clinical data in the TCGA ovarian cancer database analyzed in this study can be accessed on UCSC Xena (https://xenabrowser.net). Previous studies (and datasets) are cited at relevant places within the text as references. Gene expression datasets, GSE26193 and GSE53963, in the GEO database (https://www.ncbi.nlm.nih.gov/geo/) were used to verify the mRNA expression level of the key genes in our risk signature.

Competing interests: The authors have no other relevant affiliations or financial involvement with any organization or entity with a financial interest in or financial conflict with the subject matter or materials discussed in the manuscript apart from those disclosed.

Funding:The study was supported by 345 Talent Project of Shengjing Hospital.

Authors' contributions: MT and LZ designed research and wrote manuscript. SW, FL, HXand JG involved in data collection and data statistical analysis. MT performed the validation experiment. LZ provided fund support and critically reviewed the manuscript. All authors contributed to the article and approved the submitted version.

Acknowledgments: Thanks to all the authors who participated in writing this article.

\section{References}

1. Torre, L.A., et al., Ovarian cancer statistics, 2018. CA Cancer J Clin, 2018. 68(4): p. 284-296.

2. Tomar, T., et al., Methylome analysis of extreme chemoresponsive patients identifies novel markers of platinum sensitivity in high-grade serous ovarian cancer. BMC Med, 2017. 15(1): p. 116.

3. Armstrong, D.K., et al., NCCN Guidelines Insights: Ovarian Cancer, Version 1.2019. J Natl Compr Canc Netw, 2019. 17(8): p. 896-909.

4. Schultz, M.D., et al., Human body epigenome maps reveal noncanonical DNA methylation variation. Nature, 2015. 523(7559): p. 212-6.

5. Majumder, A., et al., Hypermethylation: Causes and Consequences in Skeletal Muscle Myopathy. J Cell Biochem, 2017. 118(8): p. 2108-2117.

6. Moufarrij, S., et al., Epigenetic therapy for ovarian cancer: promise and progress. Clin Epigenetics, 2019. 11(1): p. 7.

7. Strathdee, G., et al., Primary ovarian carcinomas display multiple methylator phenotypes involving known tumor suppressor genes. Am J Pathol, 2001. 158(3): p. 1121-7.

8. Rathi, A., et al., Methylation profiles of sporadic ovarian tumors and nonmalignant ovaries from highrisk women. Clin Cancer Res, 2002. 8(11): p. 3324-31.

9. Swisher, E.M., et al., Methylation and protein expression of DNA repair genes: association with chemotherapy exposure and survival in sporadic ovarian and peritoneal carcinomas. Mol Cancer, 
2009. 8: p. 48.

10. Ibanez de Caceres, I., et al., Tumor cell-specific BRCA1 and RASSF1A hypermethylation in serum, plasma, and peritoneal fluid from ovarian cancer patients. Cancer Res, 2004. 64(18): p. 6476-81.

11. Makarla, P.B., et al., Promoter hypermethylation profile of ovarian epithelial neoplasms. Clin Cancer Res, 2005. 11(15): p. 5365-9.

12. Tam, K.F., et al., Methylation profile in benign, borderline and malignant ovarian tumors. J Cancer Res Clin Oncol, 2007. 133(5): p. 331-41.

13. Gevaert, O., MethylMix: an R package for identifying DNA methylation-driven genes. Bioinformatics, 2015. 31(11): p. 1839-41.

14. Lv, L., et al., Methylation-Driven Genes Identified as Novel Prognostic Indicators for Thyroid Carcinoma. Front Genet, 2020. 11: p. 294.

15. Long, J., et al., DNA methylation-driven genes for constructing diagnostic, prognostic, and recurrence models for hepatocellular carcinoma. Theranostics, 2019. 9(24): p. 7251-7267.

16. Li, R., et al., Integrative analysis of DNA methylation-driven genes for the prognosis of lung squamous cell carcinoma using MethylMix. Int J Med Sci, 2020. 17(6): p. 773-786.

17. Wang, J., et al., LAT, HOXD3 and NFE2L3 identified as novel DNA methylation-driven genes and prognostic markers in human clear cell renal cell carcinoma by integrative bioinformatics approaches. J Cancer, 2019. 10(26): p. 6726-6737.

18. Pan, Y., et al., Analysis of methylation-driven genes for predicting the prognosis of patients with head and neck squamous cell carcinoma. J Cell Biochem, 2019. 120(12): p. 19482-19495.

19. Baci, D., et al., The Ovarian Cancer Tumor Immune Microenvironment (TIME) as Target for Therapy: $A$ Focus on Innate Immunity Cells as Therapeutic Effectors. Int J Mol Sci, 2020. 21(9).

20. Grabovska, Y., et al., Pediatric pan-central nervous system tumor analysis of immune-cell infiltration identifies correlates of antitumor immunity. Nat Commun, 2020. 11(1): p. 4324.

21. Mateescu, B., et al., miR-141 and miR-200a act on ovarian tumorigenesis by controlling oxidative stress response. Nat Med, 2011. 17(12): p. 1627-35.

22. Konecny, G.E., et al., Prognostic and therapeutic relevance of molecular subtypes in high-grade serous ovarian cancer. J Natl Cancer Inst, 2014. 106(10).

23. Robert, J., [Beyond the genome: Epigenome involvement in oncogenesis and cancer progression]. Bull Cancer, 2019. 106(10): p. 821-822.

24. Barton, C.A., et al., DNA methylation changes in ovarian cancer: implications for early diagnosis, prognosis and treatment. Gynecol Oncol, 2008. 109(1): p. 129-39.

25. Zheng, M., et al., Integrated multi-omics analysis of genomics, epigenomics, and transcriptomics in ovarian carcinoma. Aging (Albany NY), 2019. 11(12): p. 4198-4215.

26. Strausberg, R.L., et al., Generation and initial analysis of more than 15,000 full-length human and mouse cDNA sequences. Proc Natl Acad Sci U S A, 2002. 99(26): p. 16899-903. 
27. Parks, S.K., J. Chiche, and J. Pouyssegur, Disrupting proton dynamics and energy metabolism for cancer therapy. Nat Rev Cancer, 2013. 13(9): p. 611-23.

28. Sanhueza, C., et al., Sodium/proton exchanger isoform 1 regulates intracellular $\mathrm{pH}$ and cell proliferation in human ovarian cancer. Biochim Biophys Acta Mol Basis Dis, 2017. 1863(1): p. 81-91.

29. Wang, S., et al., Identification and characterization of MARVELD1, a novel nuclear protein that is down-regulated in multiple cancers and silenced by DNA methylation. Cancer Lett, 2009. 282(1): p. 77-86.

30. Fekete, J.T., et al., Predictive biomarkers of platinum and taxane resistance using the transcriptomic data of 1816 ovarian cancer patients. Gynecol Oncol, 2020. 156(3): p. 654-661.

31. Kobayashi, R., A. Mizutani, and H. Hidaka, Isolation and characterization of a 36-kDa microfibrilassociated glycoprotein by the newly synthesized isoquinolinesulfonamide affinity chromatography. Biochem Biophys Res Commun, 1994. 198(3): p. 1262-6.

32. Niu, D., et al., Microfibrillar-associated protein 4 (MFAP4) genes in catfish play a novel role in innate immune responses. Dev Comp Immunol, 2011. 35(5): p. 568-79.

33. Holm, A.T., et al., Characterization of spontaneous air space enlargement in mice lacking microfibrillar-associated protein 4. Am J Physiol Lung Cell Mol Physiol, 2015. 308(11): p. L1114-24.

34. Schlosser, A., et al., MFAP4 Promotes Vascular Smooth Muscle Migration, Proliferation and Accelerates Neointima Formation. Arterioscler Thromb Vasc Biol, 2016. 36(1): p. 122-33.

35. Zaravinos, A., et al., Identification of common differentially expressed genes in urinary bladder cancer. PLoS One, 2011. 6(4): p. e18135.

36. Zhao, Z., et al., microRNA-449a functions as a tumor suppressor in neuroblastoma through inducing cell differentiation and cell cycle arrest. RNA Biol, 2015. 12(5): p. 538-54.

37. Muraoka, S., et al., Strategy for SRM-based verification of biomarker candidates discovered by iTRAQ method in limited breast cancer tissue samples. J Proteome Res, 2012. 11(8): p. 4201-10.

38. Yang, J., et al., Integrated analysis of microfibrillar-associated proteins reveals MFAP4 as a novel biomarker in human cancers. Epigenomics, 2019. 11(1): p. 1635-1651.

39. Pfundt, R., et al., Constitutive and inducible expression of SKALP/elafin provides anti-elastase defense in human epithelia. J Clin Invest, 1996. 98(6): p. 1389-99.

40. Doucet, A., et al., Characterization of human pre-elafin mutants: full antipeptidase activity is essential to preserve lung tissue integrity in experimental emphysema. Biochem J, 2007. 405(3): p. 455-63.

41. Cancer Genome Atlas Research, N., Comprehensive genomic characterization defines human glioblastoma genes and core pathways. Nature, 2008. 455(7216): p. 1061-8.

42. Caruso, J.A., et al., Elafin is downregulated during breast and ovarian tumorigenesis but its residual expression predicts recurrence. Breast Cancer Res, 2014. 16(6): p. 3417.

43. Labidi-Galy, S.I., et al., Elafin drives poor outcome in high-grade serous ovarian cancers and basal-like breast tumors. Oncogene, 2015. 34(3): p. 373-83. 
44. Catron, K.M., et al., Comparison of MSX-1 and MSX-2 suggests a molecular basis for functional redundancy. Mech Dev, 1996. 55(2): p. 185-99.

45. Shames, D.S., et al., A genome-wide screen for promoter methylation in lung cancer identifies novel methylation markers for multiple malignancies. PLoS Med, 2006. 3(12): p. e486.

46. Chetcuti, A., et al., Expression profiling reveals MSX1 and EphB2 expression correlates with the invasion capacity of Wilms tumors. Pediatr Blood Cancer, 2011. 57(6): p. 950-7.

47. Wang, T., Y. Xu, and P. Hou, Identifying novel biomarkers of gastric cancer through integration analysis of single nucleotide polymorphisms and gene expression profile. Int J Biol Markers, 2015. 30(3): p. e321-6.

48. Dunwell, T.L., et al., Epigenetic analysis of childhood acute lymphoblastic leukemia. Epigenetics, 2009. 4(3): p. 185-93.

49. Yue, Y., et al., Homeobox protein MSX1 inhibits the growth and metastasis of breast cancer cells and is frequently silenced by promoter methylation. Int J Mol Med, 2018. 41(5): p. 2986-2996.

50. Yue, Y., et al., MSX1 induces G0/G1 arrest and apoptosis by suppressing Notch signaling and is frequently methylated in cervical cancer. Onco Targets Ther, 2018. 11: p. 4769-4780.

51. Bonito, N.A., et al., Epigenetic Regulation of the Homeobox Gene MSX1 Associates with PlatinumResistant Disease in High-Grade Serous Epithelial Ovarian Cancer. Clin Cancer Res, 2016. 22(12): p. 3097-3104.

52. Park, S., S. Cheon, and D. Cho, The dual effects of interleukin-18 in tumor progression. Cell Mol Immunol, 2007. 4(5): p. 329-35.

53. Dinarello, C.A. and G. Fantuzzi, Interleukin-18 and host defense against infection. J Infect Dis, 2003. 187 Suppl 2: p. S370-84.

54. Carbotti, G., et al., The IL-18 antagonist IL-18-binding protein is produced in the human ovarian cancer microenvironment. Clin Cancer Res, 2013. 19(17): p. 4611-20.

55. Medina, L., et al., Expression of IL-18, IL-18 binding protein, and IL-18 receptor by normal and cancerous human ovarian tissues: possible implication of IL-18 in the pathogenesis of ovarian carcinoma. Mediators Inflamm, 2014. 2014: p. 914954.

56. Zhu, Y. and K. Sundfeldt, Tight junction formation in epithelial ovarian adenocarcinoma. Acta Obstet Gynecol Scand, 2007. 86(8): p. 1011-9.

57. Groeneweg, J.W., et al., Notch signaling in serous ovarian cancer. J Ovarian Res, 2014. 7: p. 95.

58. Hynes, N.E. and G. MacDonald, ErbB receptors and signaling pathways in cancer. Curr Opin Cell Biol, 2009. 21(2): p. 177-84.

59. Eke, I. and N. Cordes, Focal adhesion signaling and therapy resistance in cancer. Semin Cancer Biol, 2015. 31: p. 65-75.

60. Cornel, A.M., I.L. Mimpen, and S. Nierkens, MHC Class I Downregulation in Cancer: Underlying Mechanisms and Potential Targets for Cancer Immunotherapy. Cancers (Basel), 2020. 12(7). 
61. Webb, J.R., et al., PD-L1 expression is associated with tumor-infiltrating $T$ cells and favorable prognosis in high-grade serous ovarian cancer. Gynecol Oncol, 2016. 141(2): p. 293-302.

62. Anderson, A.C., N. Joller, and V.K. Kuchroo, Lag-3, Tim-3, and TIGIT: Co-inhibitory Receptors with Specialized Functions in Immune Regulation. Immunity, 2016. 44(5): p. 989-1004.

63. Triebel, F., et al., LAG-3, a novel lymphocyte activation gene closely related to CD4. J Exp Med, 1990. 171(5): p. 1393-405.

64. Fucikova, J., et al., TIM-3 Dictates Functional Orientation of the Immune Infiltrate in Ovarian Cancer. Clin Cancer Res, 2019. 25(15): p. 4820-4831.

65. Ganesh, K. and J. Massague, Targeting metastatic cancer. Nat Med, 2021. 27(1): p. 34-44.

\section{Figures}

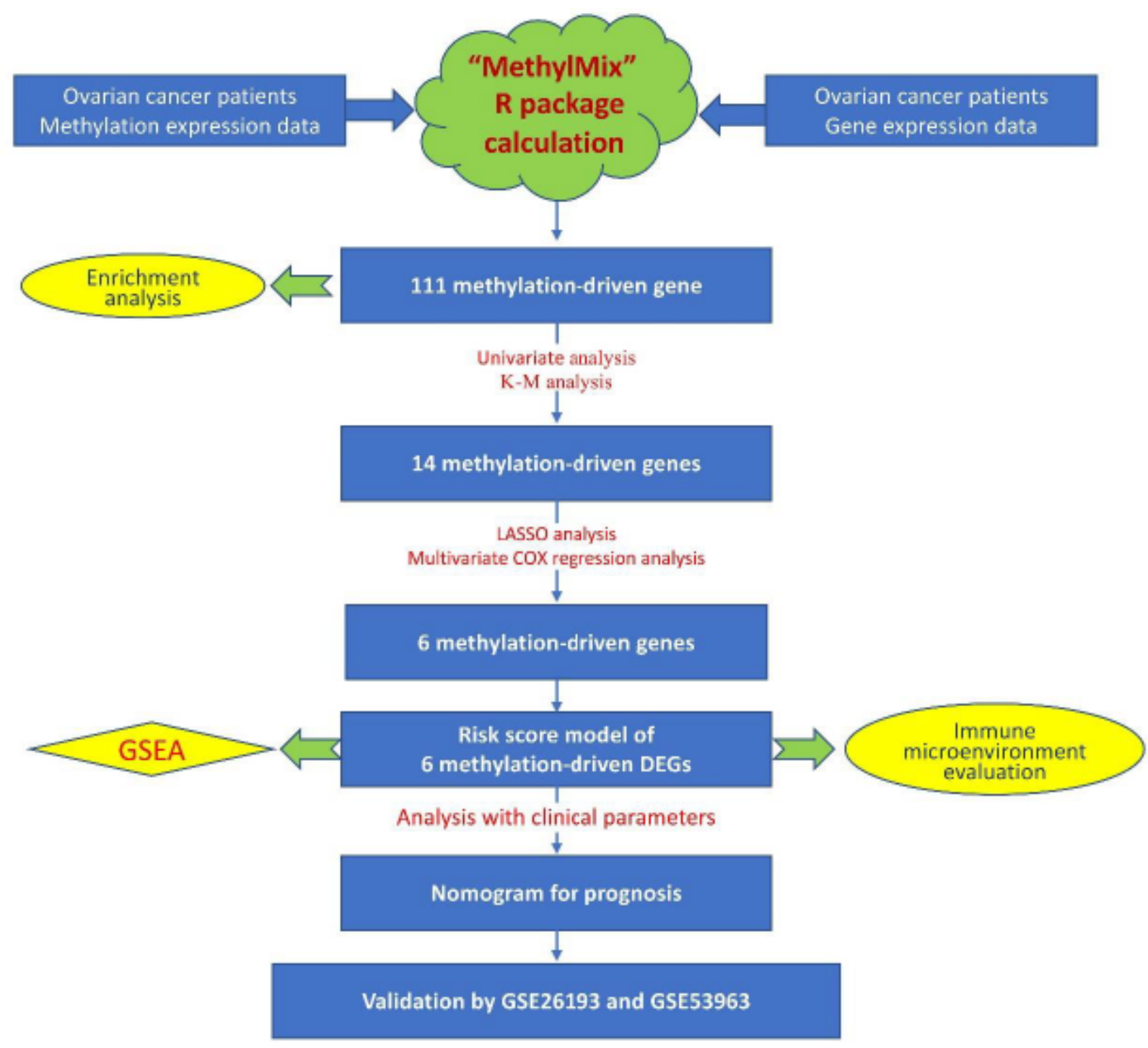

\section{Figure 1}

The study workflow illustrating the identification and validation of the DNA MDGs in EOC. 


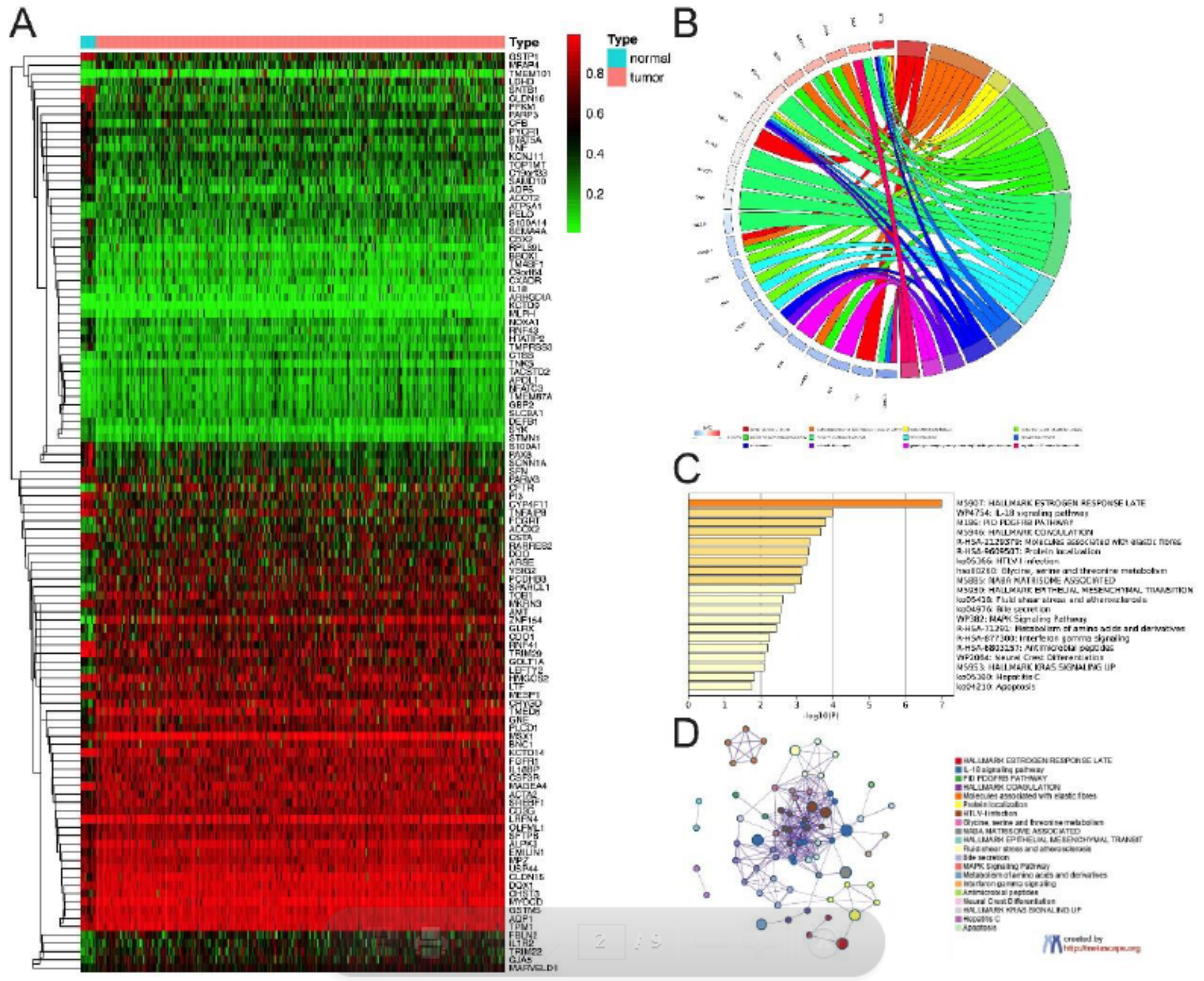

Figure 2

Functional exploration of candidate DNA MDGs (A) Heatmap of the candidate DNA MDGs $(n=111)$ in EOC. (B) The circle represents the correlation between 22 MDGs and their GO terms. (C) Heatmap of top 20 GO pathways. (D) Enriched ontology clusters colored by cluster-ID 

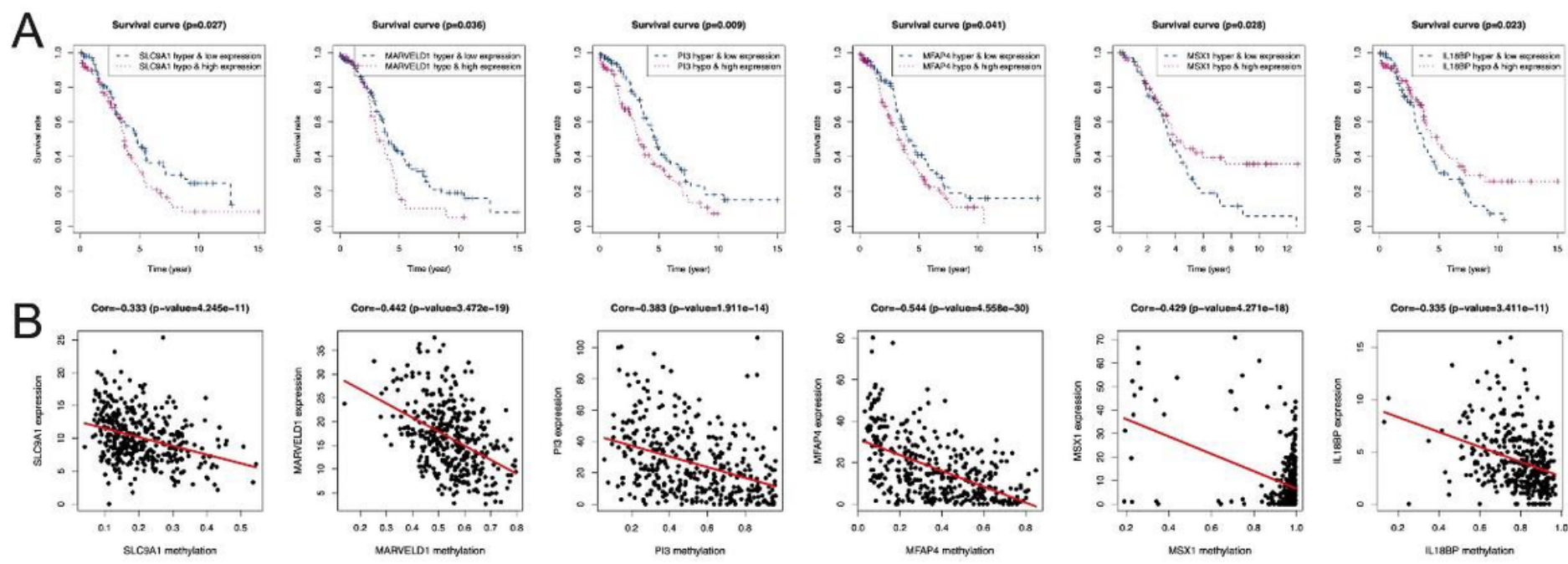

C
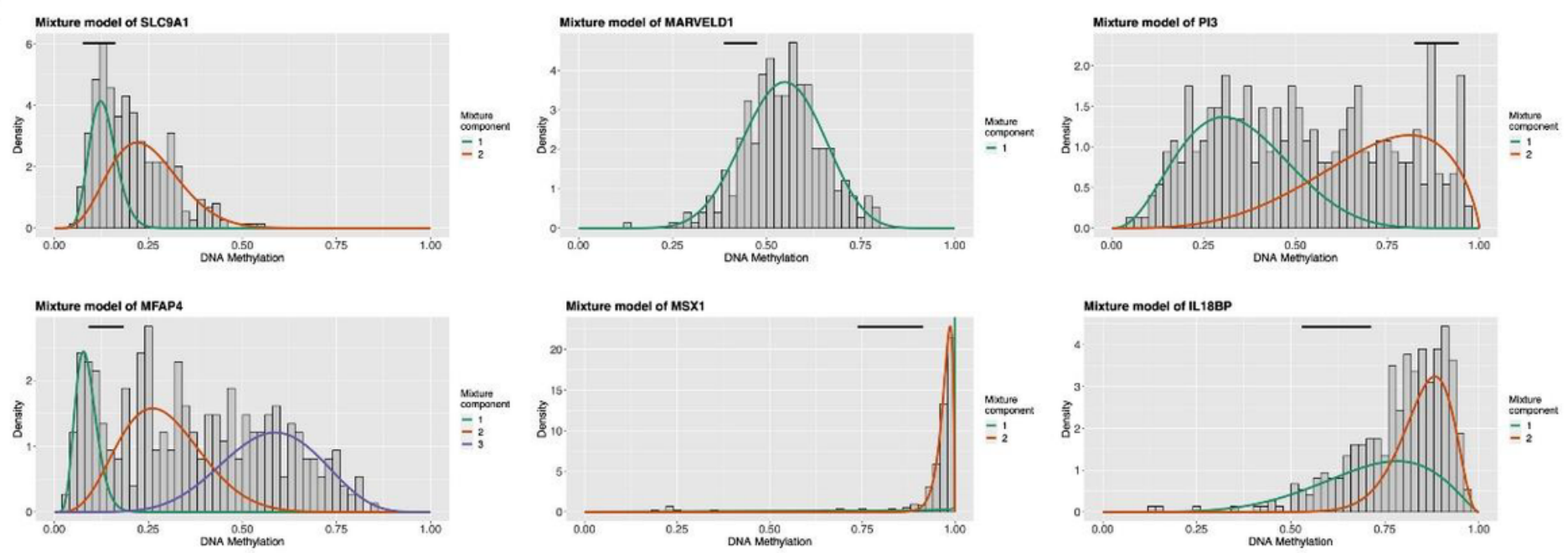

Figure 3

DNA MDGs. (A) KM analysis of gene expression level and DNA methylation level for the six DNA MDGs. (B) Regression analysis between the mRNA expression level and DNA methylation degree of the six DNA MDGs. (C) The histogram demonstrates the distribution of SLC9A1区MARVELD1, PI3, MFAP4, MSX1, IL18BP methylation in EOC samples. 
A

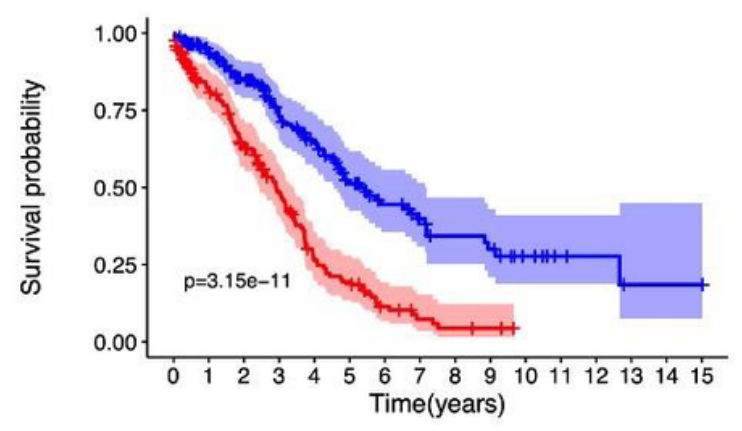

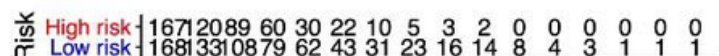
$\begin{array}{llllllllllllllll}0 & 1 & 2 & 3 & 4 & 5 & 6 & 7 & 8 & 9 & 10 & 11 & 12 & 13 & 14 & 15\end{array}$ Time(years)

$\mathrm{C}$

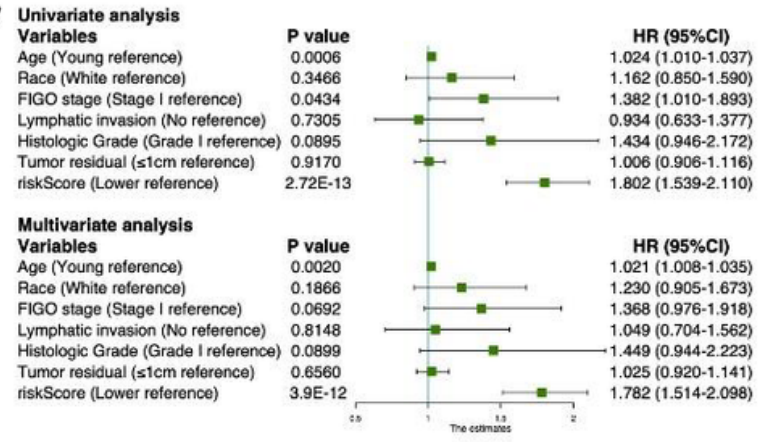

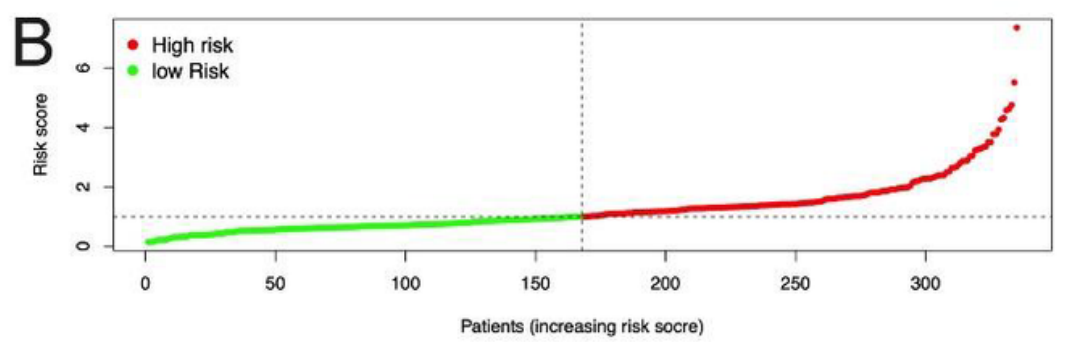
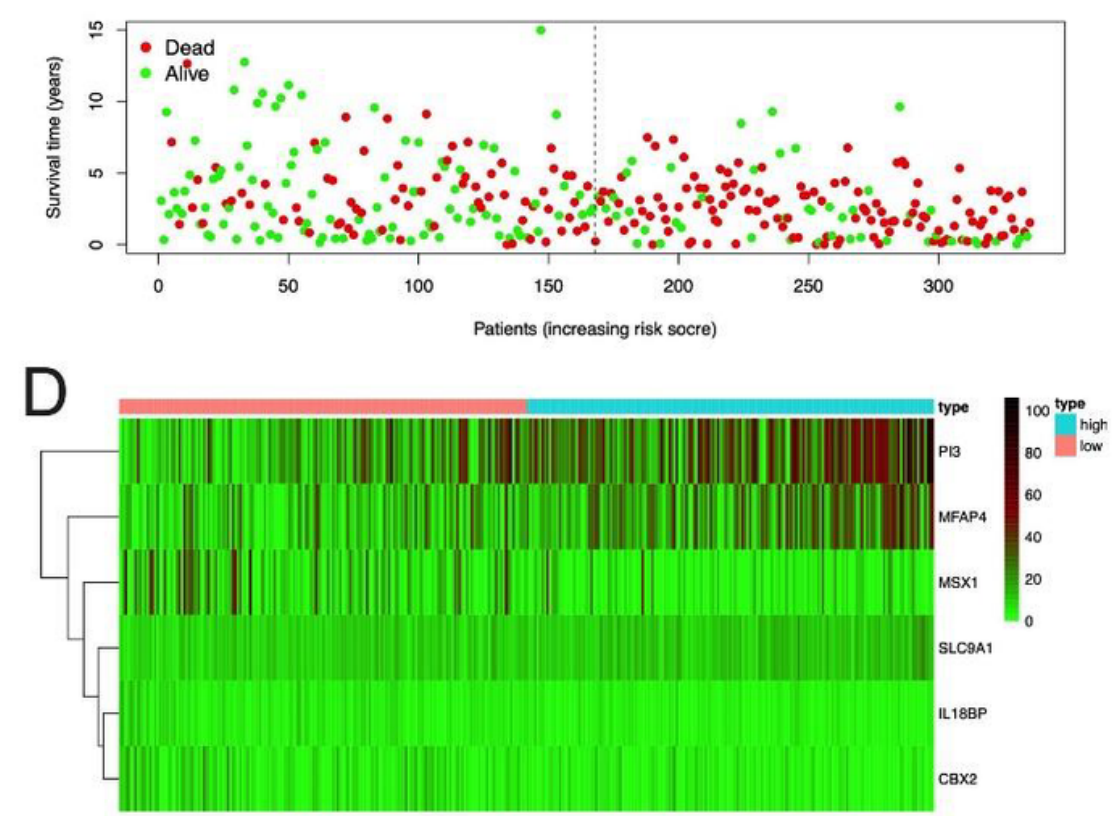

\section{Figure 4}

The risk score model is based on the six MDGs. (A) KM analysis of the OS between the two groups. (B) the distributions of the risk score and survival status of the patients. (C) Univariate and multivariate analyses of age, race, FIGO stage, lymphatic invasion, histologic grade, tumor residual, and risk score with OS. (D)The 6 gene expression data and distribution of risk scores between the two groups in the UCSC Xena website database were visualized via a heatmap. 

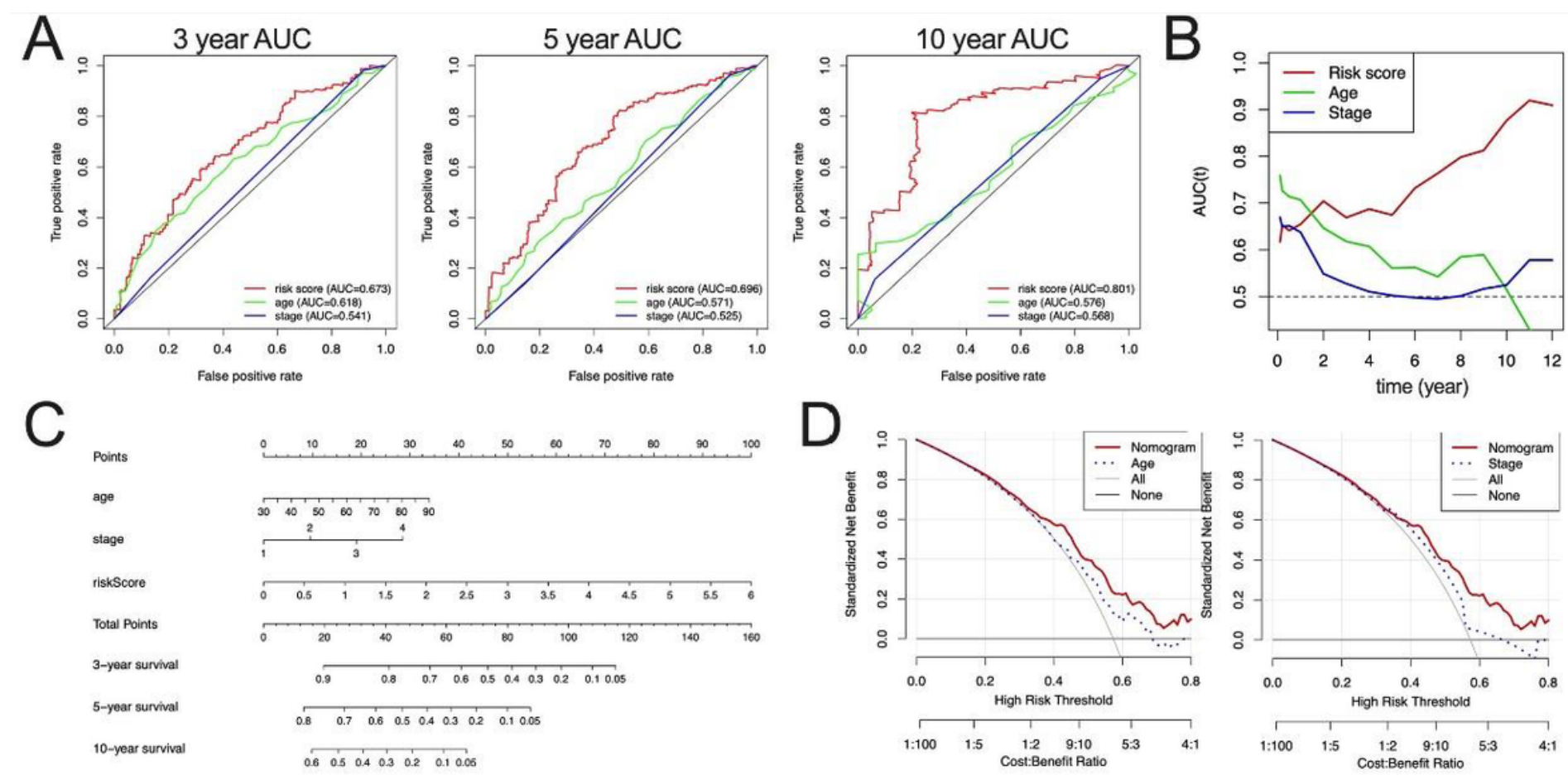

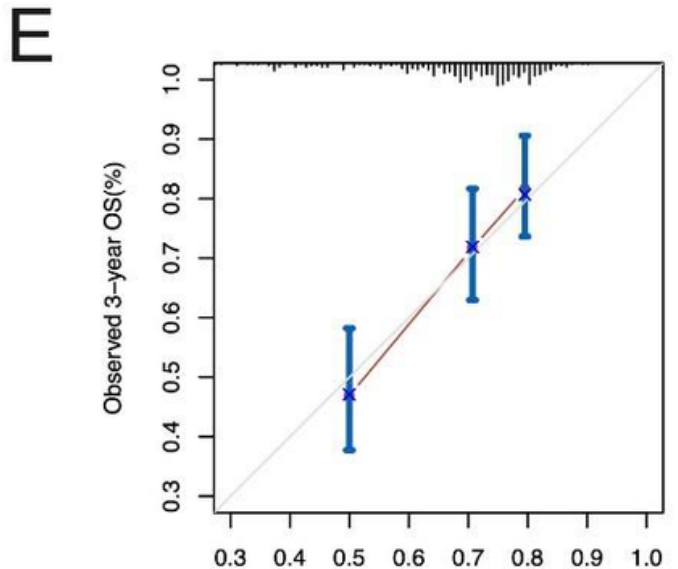

Nomogram-Predicted 3-year OS (\%)

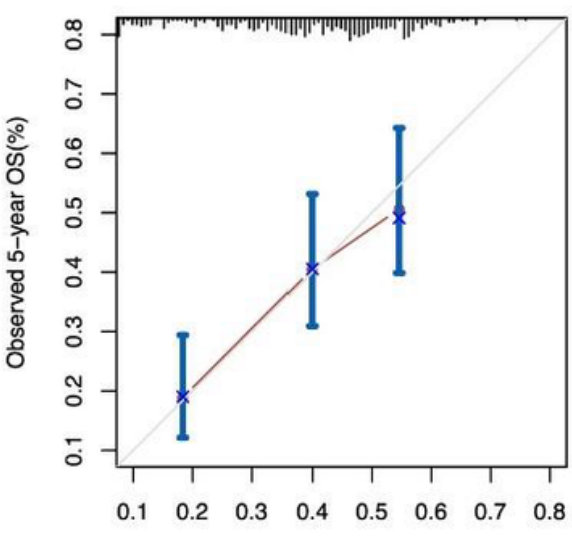

Nomogram-Predicted 5-year OS (\%)

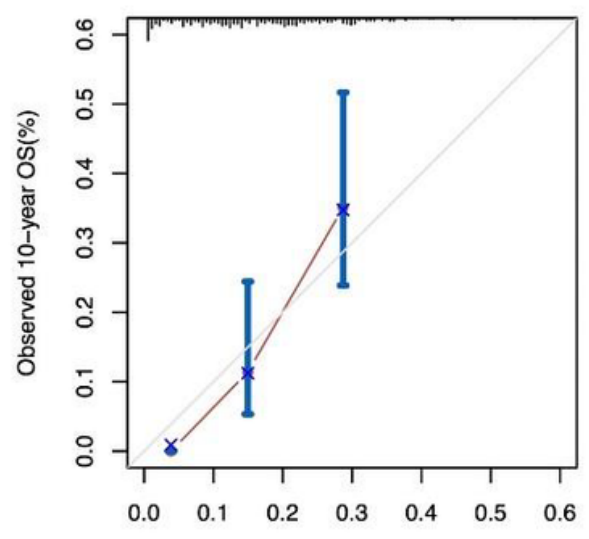

Nomogram-Predicted 10-year OS (\%)

Figure 5

Evaluation of the risk score for OS prediction in EOC. (A) The accuracy of the OS risk score model evaluated by AUC for 3-, 5-and 10-year survival times. (B) Time-dependent AUC of the risk score, age and FIGO stage to predict the OS probability within 12 years. (C) The constructed nomogram for predicting the 3-, 5- and 10-year OS of EOC patients. (D) The clinical model was assessed by DCA. The X-axis is the probability of predicting OS, while the Y-axis is the standardized net Benefit. The DCA of the nomogram was found to be significantly higher than that of age and FIGO staging. (E) Calibration curves of the nomogram at 3-, 5- and 10-year OS. 
A

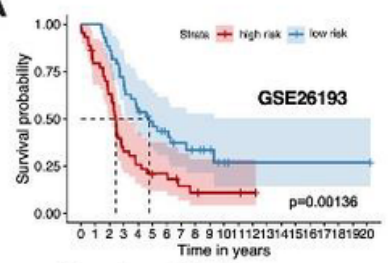

Percentage at risk (\%):

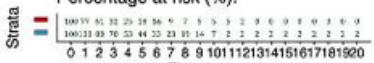
Time in years

$\mathrm{D}$

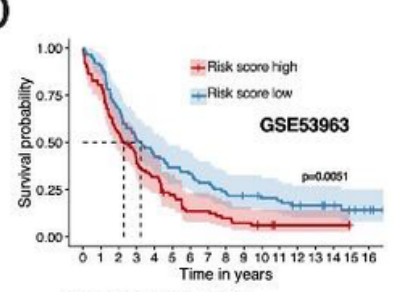

Percentage at risk (\%):
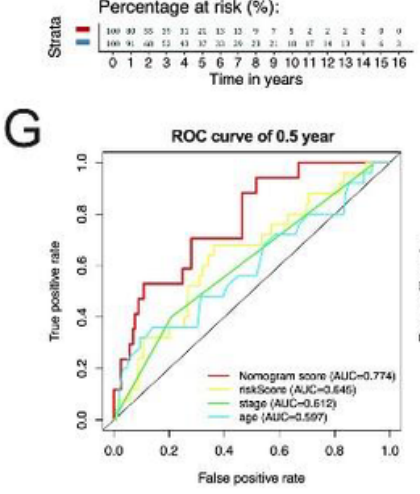
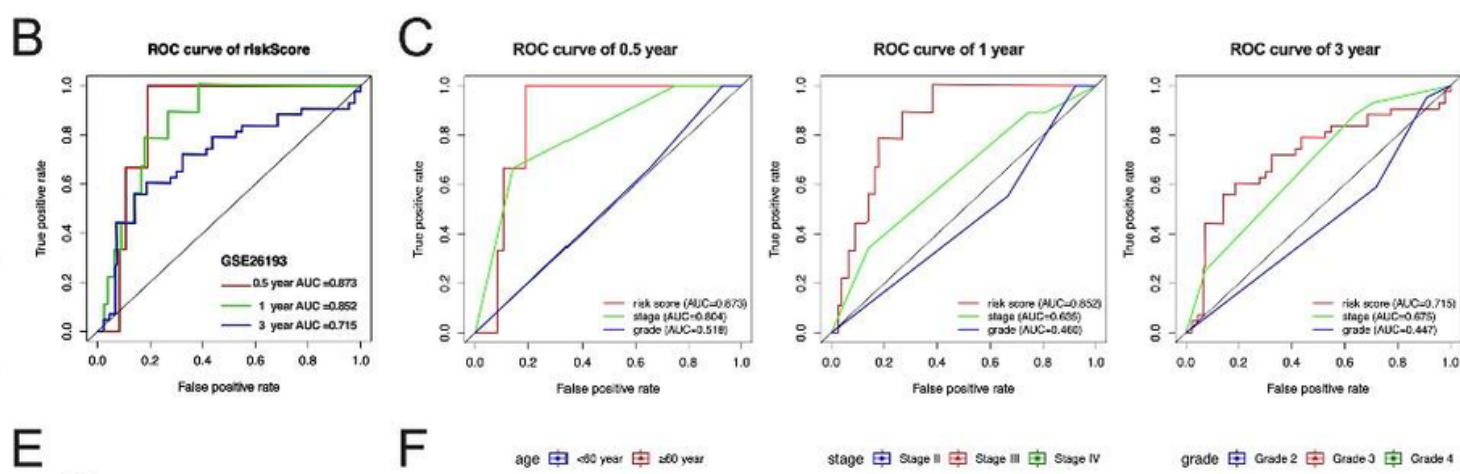

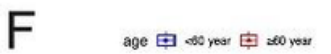
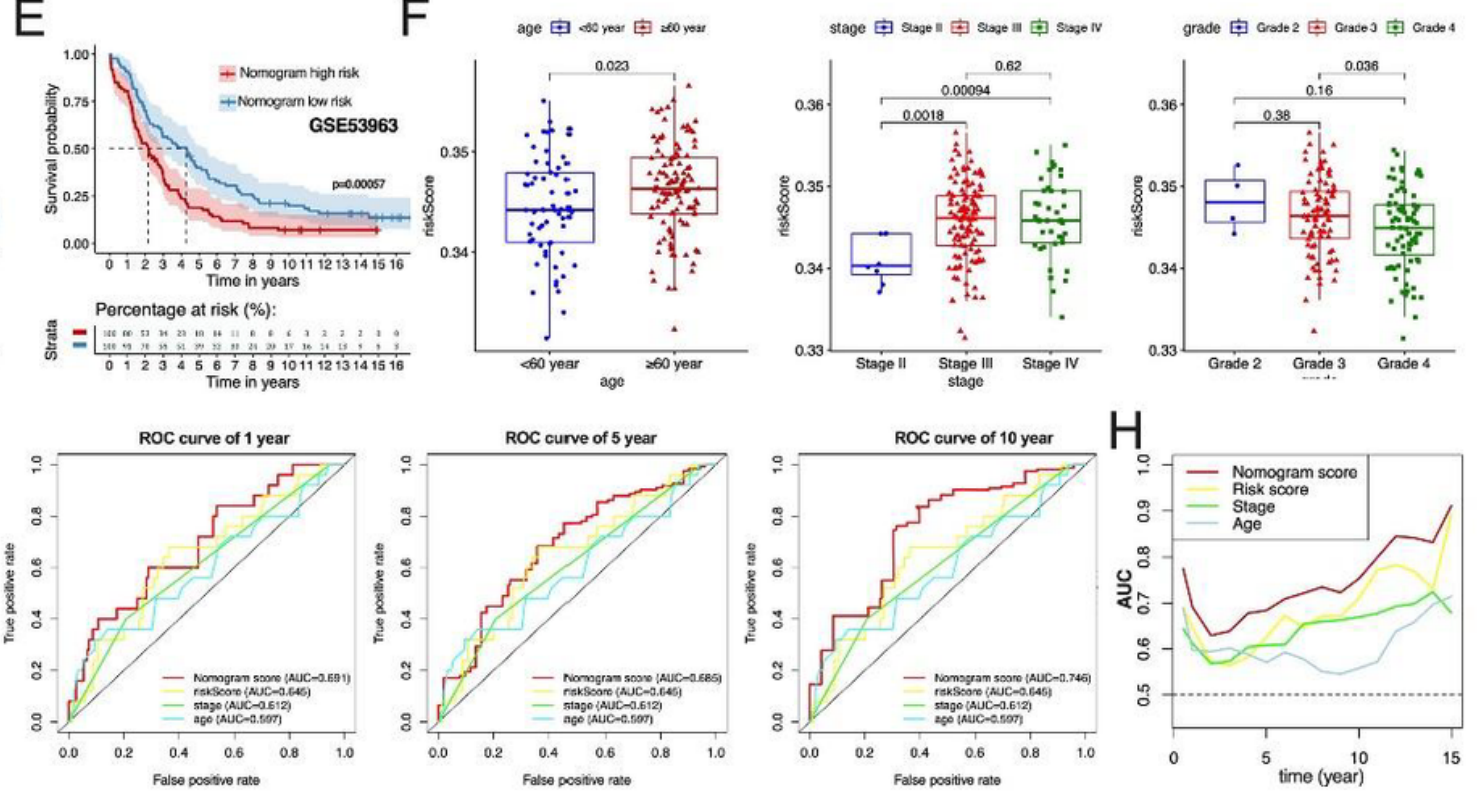

Figure 6

External Validation in GEO datasets, GSE26193 and GSE53963. GSE26193 dataset: (A) KM analysis of the two risk score groups. (B) The AUC of the survival prediction risk score for 0.5-, 1-and 3-year survival times. (C) The AUC of the survival prediction risk score, age, and FIGO stage in the validation dataset for 0.5-, 1-and 3-year survival times. GSE53963 database: (D) KM analysis of OS between the two risk score groups (E) KM analysis of OS between the two nomogram groups. (F) The relationship between the risk score and age, FIGO stage, and histologic grade. (G) The AUC of the survival prediction risk score, nomogram, age, and FIGO stage in the validation dataset for 0.5-, 1-, 5- and 10-year survival times. $(\mathrm{H})$ Time-dependent AUC for the prediction of OS within 15 years. 

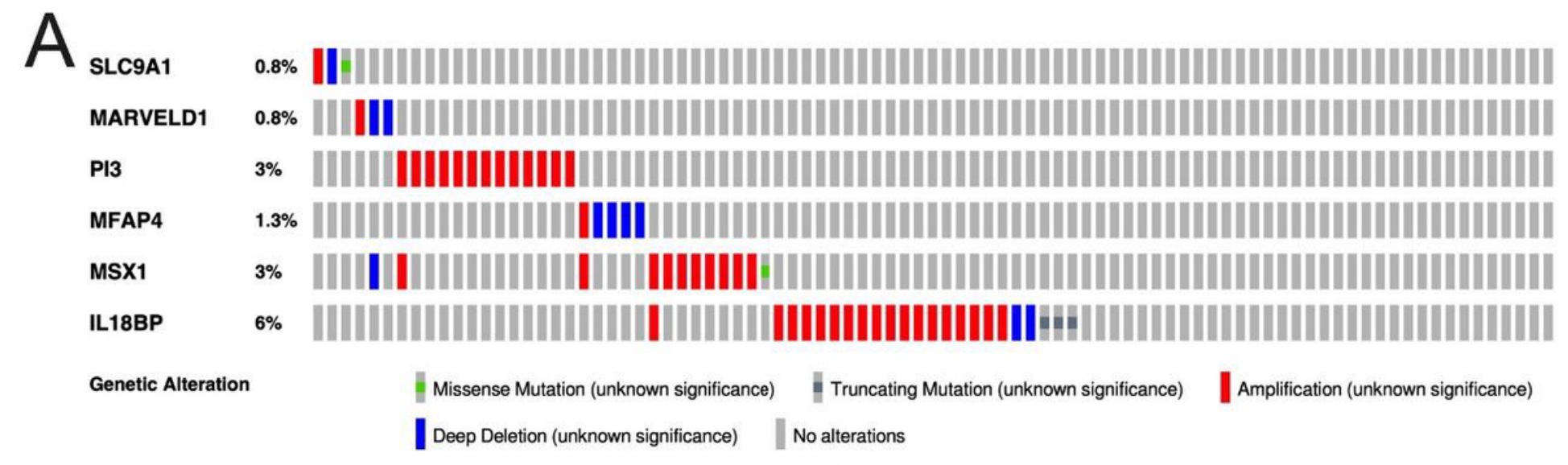

B
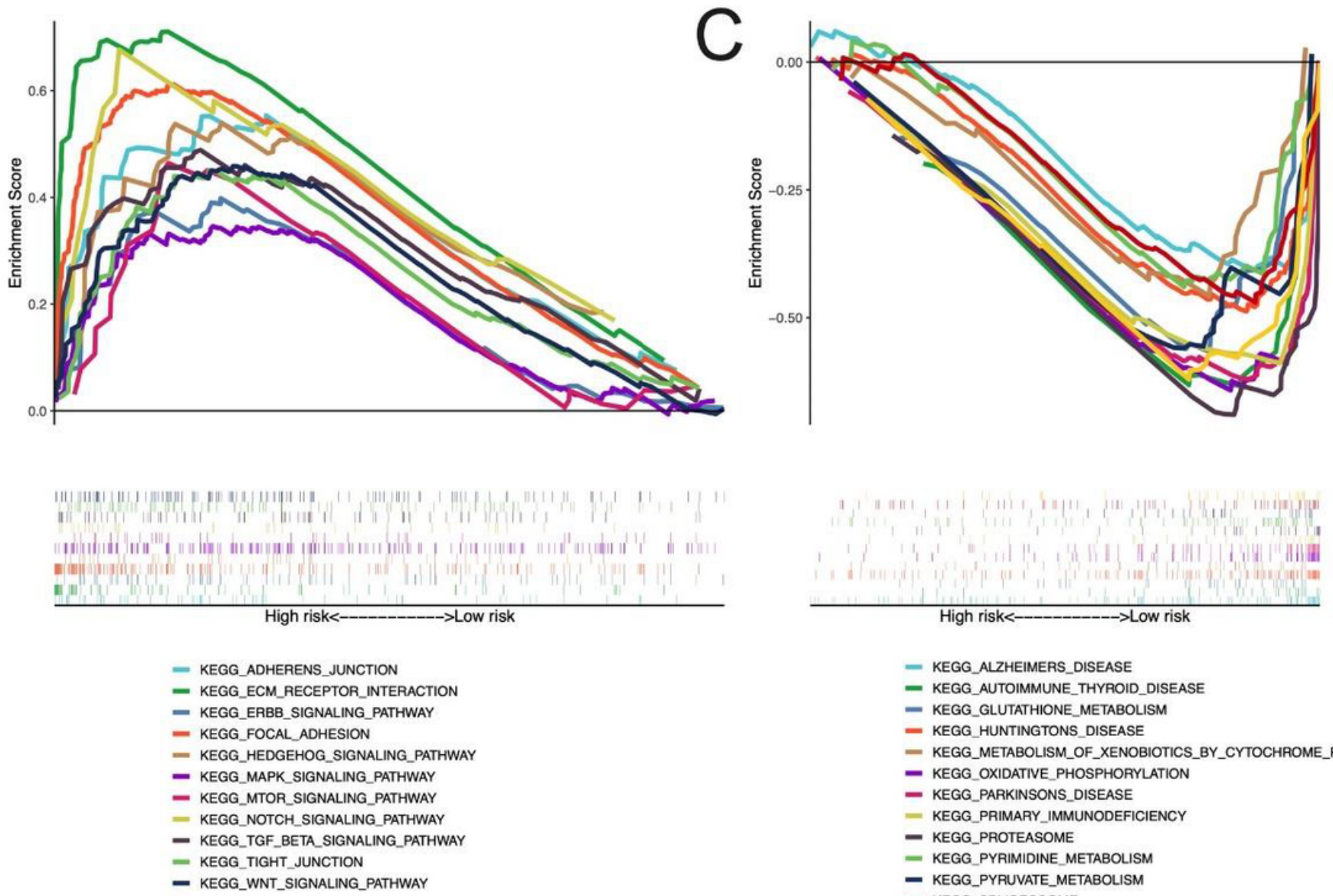
- KEGG_ALZHEIMERS_DISEASE
- KEgG_AUTOIMMUNE_THYROID_DISEASE
- KEGG_GLUTATHIONE_METABOLISM
- KEGG_HUNTINGTONS_DISEASE
- KEGG_METABOLISM_OF_XENOBIOTICS_BY_CYTOCHROME_P450
- KEGG_OXIDATIVE_PHOSPHORYLATION
- KEGG_PARKINSONS_DISEASE
- KEGG_PRIMARY_IMMUNODEFICIENCY
- KEGG_PROTEASOME
- KEGG_PYRIMIDINE METABOLISM
- KEgG_PYRUVATE_METABOLISM
- KEGG_SPLICEOSOME
- KEGG_TYPE_I_DIABETES_MELLITUS

Figure 7

CNV and GSEA analysis of DNA MDGs in the risk score model. (A) CNV analysis of DNA MDGs in EOC samples. (B) Enrichment plots of the top ten enrichment pathways in the high-risk score group. (C) Enrichment plots of the top ten enrichment pathways in the low-risk score group. 

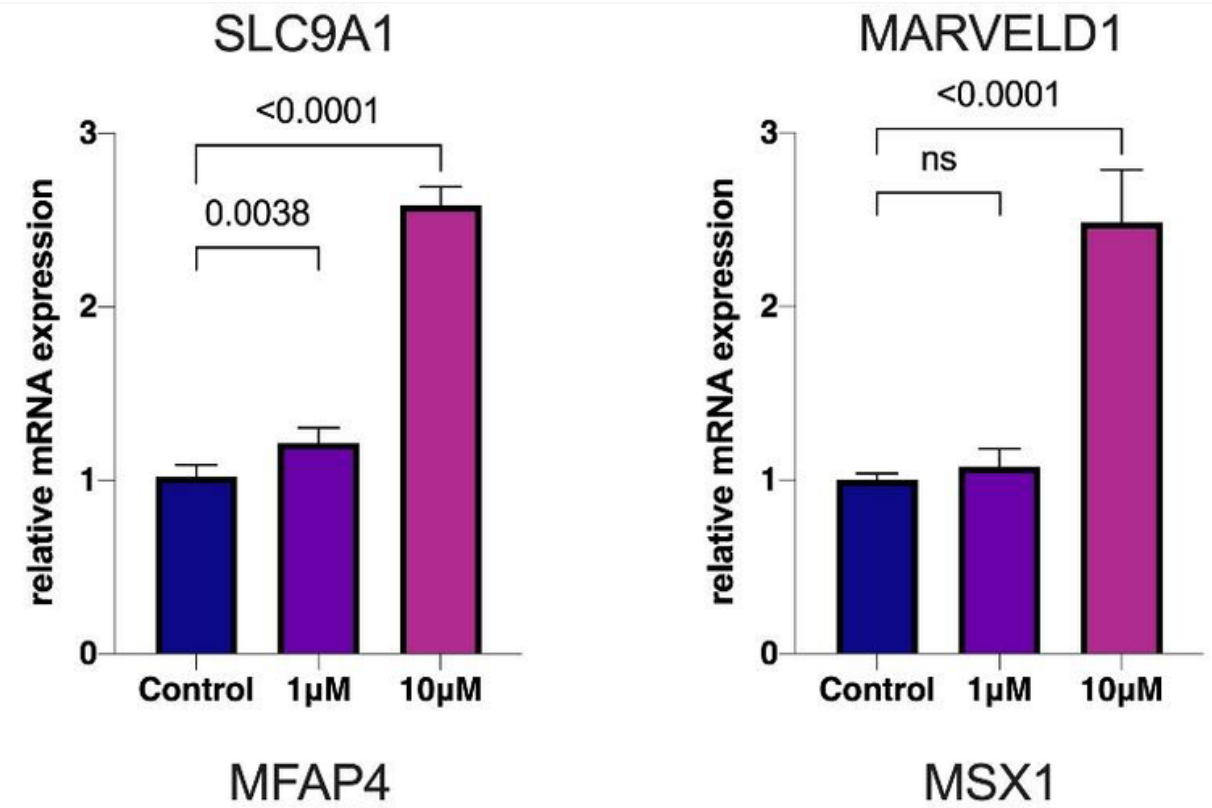

PI3
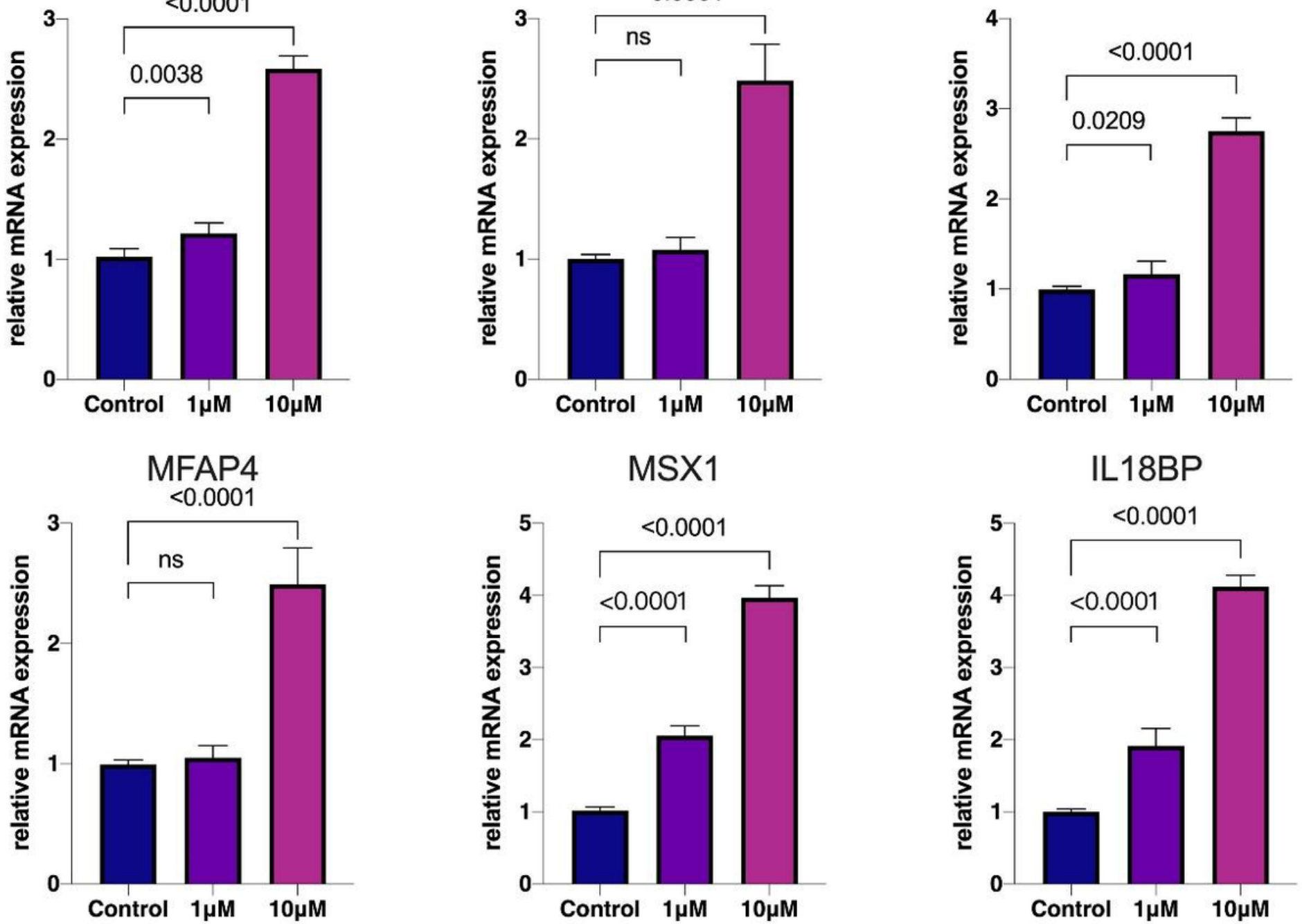

Figure 8

mRNA expression of the six genes in OVCAR3 cells after DAC (1 $\mu$ l and 10 $\mu$ l) treatment. (A) SLC9A1 mRNA expression. (B) MARVELD1 mRNA expression. (C) PI3 mRNA expression. (D) MFAP4 mRNA expression. (E) MSX1 mRNA expression. (F) IL18BP mRNA expression. 
A

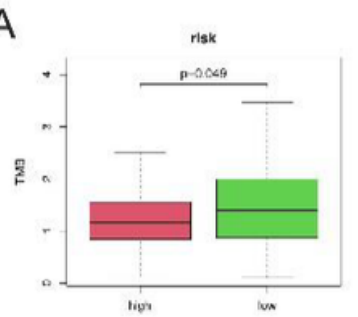

B

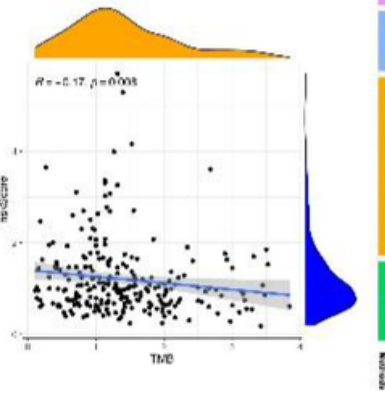

E Immune function

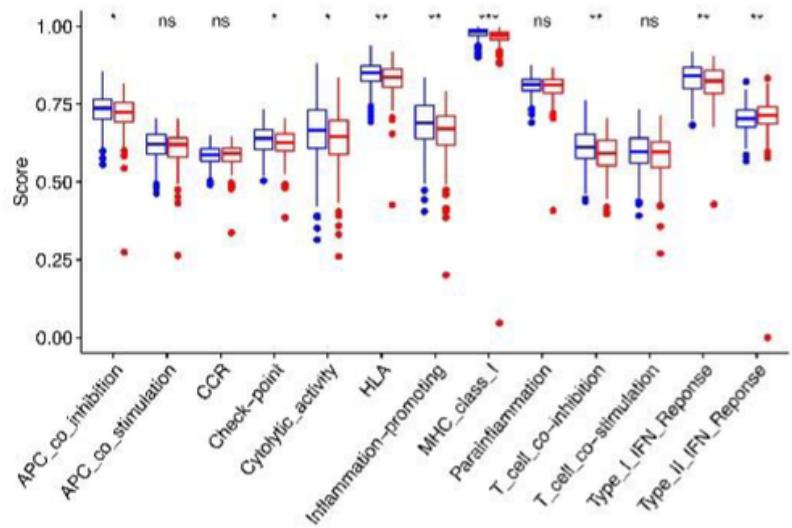

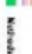

C

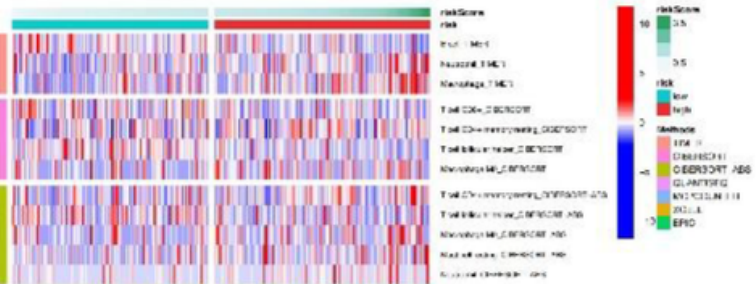

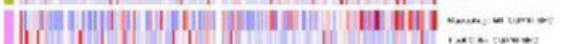

| || || || || || || || || || ||||| || || ||| ||

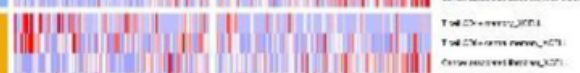

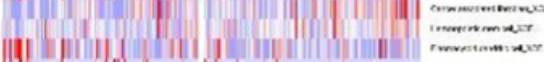

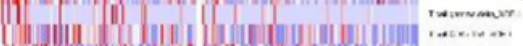

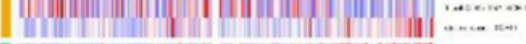

(1)

insersc
D
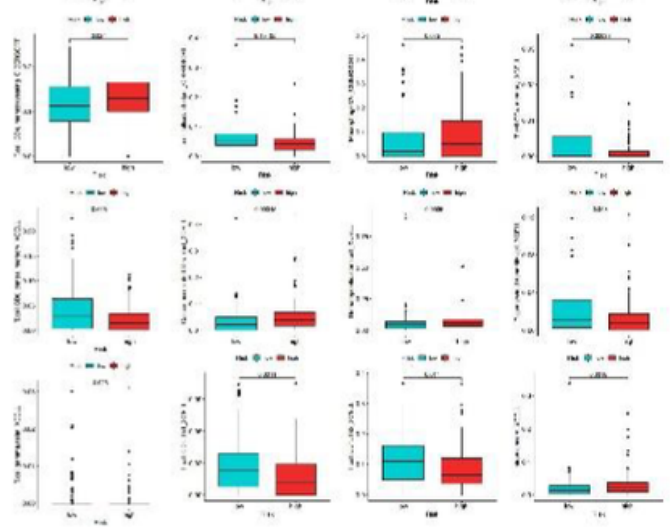

Check point Risk low high

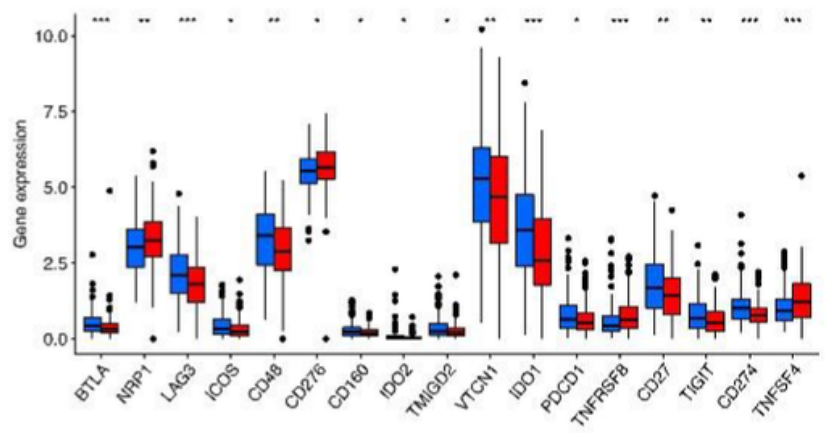

Figure 9

Immune assessment of two risk score model groups (A) (B) TMB analysis of the two risk score groups. (C)Heatmap of the TIMER, CIBERSORT, CIBERSORT-ABS, QUANTISEQ, MCPCOUNTER, XCELL, EPIC analysis of the two risk score groups. (D) 16 groups with significant differential expression from heatmap. Shows the correlation between immune infiltration, immune cell distribution and the two risk score groups. (E) Immune function analysis of the two risk score groups. (F) Check point analysis of the two risk score groups.

\section{Supplementary Files}

This is a list of supplementary files associated with this preprint. Click to download.

- supplementaryfiguresandtables.docx 